\title{
Geschätzter Werkstoff ist jetzt auch chairside einsetzbar
}

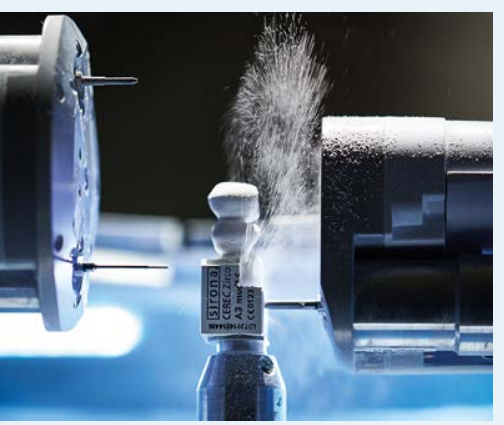

CEREC bietet jetzt in der Zahnarztpraxis einen völlig neuen Prozess: Durch die Kombination des neuen CEREC SpeedfireOfens mit CEREC Zirconia können Vollkronen und Brücken aus hochwertigem Zirkonoxid in der Praxis angefertigt werden, während der Patient wartet. Das Material eignet sich sowohl für Einzelkronen als auch für kleine Brücken und lässt sich in dünnen Wandstärken verarbeiten. Da diese Restaurationen in einer monolithischen Form hergestellt werden, besteht kein Risiko für Chipping. Ein weiterer Vorteil für den Behandler: Zirkonoxid kann konventionell zementiert werden. Das Material ist ein voreingefärbtes transluzentes Zirkonoxid, das in 10 Farben in Anlehnung an den VITA Classic Shade Guide erhältlich ist. Es wird vergrößert gefräst und anschließend im neuen Sinterofen CEREC SpeedFire auf seine endgültige Größe dichtgesintert. Das übergroße Schleifen fördert eine neue Dimension der Schleifgenauigkeit, was zu passgenauen Restaurationen führt. Der Sinterprozess benötigt für Kronen 10-15 min und für Brücken 25 min. Ein anschließender Glasurbrand bringt die Restauration auf Hochglanz. Der Arbeitsablauf ist einfach zu erlernen, da die CEREC Software 4.4.1 den Zahnarzt durch den gesamten Prozess führt und sogar die Sinter- und Glasierinformationen an den Ofen übermittelt. Es ist keine Programmierung des Ofens erforderlich - das wird alles automatisch von der Software übernommen. Für einen einfachen Prozess und eine hohe Versorgungsqualität sorgen ein Hochleistungswerkstoff und ein speziell auf das Material abgestimmter Workflow. Ausführliche Informationen zu CEREC Zirconia finden Zahnärzte auf der neu gestalteten Website http://www.cerec.com/Zirkonoxid.

Nach einer Pressemitteilung der Sirona Dental GmbH, A - Wals bei Salzburg 